\title{
Editorial
}

\section{Improving outcome for scirrhous gastric cancer}

\author{
H.H. HARTGRINK \\ Department of Surgery, Leiden University Medical Centre, PO Box 9600, 2300 RC Leiden, The Netherlands
}

Gastric cancer is still the second most common cause of cancer death in the world. Survival is related to the stage of the disease at diagnosis and to the histological profile. From Japanese registry data it was shown that about $11 \%$ of all gastric cancers are scirrhous [1]. Together with lymphatic invasive forms of gastric cancer, they lead to metastasis even in earlier stages and therefore have poor prognosis. Improvement of survival could be achieved through prevention, early detection, and optimization of treatment.

Prevention of gastric cancer seems to be the most logical approach to decrease cancer-related death. The exact cause of gastric cancer, however, is not always known. Epidemiologic studies show that smoking, food preservation, and low consumption of fresh fruit and vegetables are associated with an increased gastric cancer risk [2]. In addition, Helicobacter pylori is a major risk factor for the development of gastric cancer [3]. About $50 \%$ of the world population is affected with this bacterium. Although eradication of $H$. pylori has shown a beneficial effect in experimental studies, metaanalyses have not found a significant benefit for human beings [4]. Only after endoscopic mucosal resection for early gastric cancer, eradication of $H$. pylori proved beneficial to prevent metachronic gastric cancer [5].

Early detection is another approach to improve outcome. In East Asian countries the incidence of gastric cancer is high, and in these countries screening is cost-effective. In almost all other countries in the world there is insufficient clinical evidence to recommend population-based endoscopic screening [6]. Despite screening, it is difficult to identify scirrhous gastric cancer in an early stage. Owing to its submucosal diffuse growth form, endoscopic appearance often underestimates the stage of disease.

Offprint requests to: H.H. Hartgrink
The curative treatment of gastric cancer has been basically only surgical. Many trials and comparative studies have tried to find out to what extent surgery should be performed. There is a great difference between results from the East and the West. Comparative studies from Japan in the early 1990s showed an improved survival for D2 lymph node dissections over D1 and D0 dissections. In the Dutch gastric cancer and the British MRC gastric cancer randomized trials no significant difference was found between D1 and D2 lymph node dissections [7, 8]. It was suggested that the higher morbidity and mortality for D2 dissections probably had offset the possible benefit in survival. Indeed, a subgroup analysis of patients who did not have a pancreatico-splenectomy showed a significant survival benefit after a D2 lymph node dissection over a D1.

A more recent randomized trial from Taiwan by $\mathrm{Wu}$ et al. did show a significant survival benefit after a D3 lymph node dissection [9]. This result could probably be reached because there was no mortality in either arm; therefore, the theoretical benefit of an extended lymph node dissection does become evident if mortality can be avoided. However, Sasako et al. found no difference in survival for a D2 dissection and a D2 dissection with para-aortal lymph node dissection in a large randomized trial in Japan [10]. The extent of surgery therefore seems to have reached its limits. The results for gastric cancer surgery remain poor especially for the more advanced stages.

To further improve outcome, (neo-)adjuvant treatment with radiotherapy and/or chemotherapy has been tested in many regimens. Radiotherapy alone in an adjuvant setting has a modest favorable impact on locoregional control, but survival benefit has not been proven. Radiotherapy preceding surgery, however, did show a significant improvement in 3-and 5-year survival in a meta-analysis by Fiorica et al. [11]. Compliance of radiotherapy was $100 \%$ and there was no increase in anastomotic leakage or mortality. Despite these results 
more studies on the subject are unlikely to be conducted, as all efforts are currently directed toward postoperative radiochemotherapy and perioperative chemotherapy.

Postoperative radiochemotherapy has been shown beneficial in the Southwest Oncology Group (SWOG)Intergroup 0116 trial and is now standard treatment in the United States [12]. One of the main criticisms of this study is that most patients had a D0 dissection instead of the recommended $\mathrm{D} 2$ dissection, and so this adjuvant treatment may have compensated for inadequate surgery. Indeed, a subgroup analysis showed no effect in the D2 dissection group.

A Korean observational study by Kim et al., however, did show a clinical benefit of postoperative radiochemotherapy in a population of more than 500 patients after gastric cancer resection with a D2 lymph node dissection [13].

Neo-adjuvant radiochemotherapy has been tested in several small phase I and II trials. In these trials, partial/ complete response rates were between $35 \%$ and $65 \%$, and toxicity was acceptable. Conceptually, neoadjuvant radiochemotherapy therefore unifies the benefit of radiochemotherapy with the advantages of a neo-adjuvant approach and should be further explored in clinical phase III trials.

Adjuvant chemotherapy has been tested in many trials and regimens. Several meta-analyses on this subject have shown no or a modest significant benefit of $3 \%-5 \%$, but this benefit is considered too small to justify adjuvant chemotherapy. The most effective regimens nowadays seem to consist of fluorouracil, cisplatin, and an anthracycline, although the REAL-2 study showed that fluorouracil can be replaced by capecetabine, and cisplatin by oxaliplatin.

One of the few positive Western studies on chemotherapy is the MAGIC study, which randomly compared subjects who had undergone only surgery for advanced resectable gastric cancer with those who also had received perioperative chemotherapy with three courses of epirubicin, cisplatin, and fluorouracil (ECF) pre- and postoperatively [14]. After a median follow-up of 4 years, the group with perioperative chemotherapy had an improved 5-year overall (36\% vs $23 \% ; P=0.009)$ and progression-free survival $(P<0.001)$. However, only $42 \%$ of all patients completed the postoperative chemotherapy. With the poor results of adjuvant chemotherapy in mind, the positive overall results of the MAGIC study are probably due mainly to the preoperative chemotherapy.

Neo-adjuvant treatment was also tested in the randomized Dutch FAMTX trial with the use of 5-FU, adriamycin, and methotrexate, but results were negative [15]. At the time, FAMTX was the most effective regimen but obviously was not effective enough. With the use of 5-FU and cisplatin, recently Boige et al. did find an advantage in disease-free and overall survival (OS) for neo-adjuvant chemotherapy (5-year OS, 38\% vs $24 \% ; P=0.02$ ) [16].

In the MAGIC trial as well as the French trial by Boige et al., neo-adjuvant treatment led to a decreased tumor size, while morbidity was acceptable.

For Eastern patients adjuvant chemotherapy with S1, an oral fluoropyrimidine, has been proven to be beneficial [17]. Survival after a D2 dissection was $70.1 \%$ in the surgery only group and $80.1 \%$ in the adjuvant $\mathrm{S} 1$ group $(P=0.002)$. For Western patients this drug in its current form causes severe diarrhea. Adjusted forms are being developed and tested.

With S1 being effective in the adjuvant setting it is only logical that the drug S1 has been tested in a neoadjuvant setting. The phase II study by Kinoshita et al. published in this issue of Gastric Cancer has shown that toxicity of S1 was acceptable and the postoperative morbidity was not increased by the neo-adjuvant treatment [18]. However, the response rate of $32.6 \%$ obviously was not enough to improve outcome and therefore it was concluded that neo-adjuvant $\mathrm{S} 1$ alone will not be tested in a phase III trial. Fortunately, more effective regimens such as the combination of $\mathrm{S} 1$ with cisplatin are now available, and response rates of up to $76 \%$ have been reported. With such a high response rate a phase III trial is feasible. Such a trial is currently under way in Japan (JCOG 0501).

One of the main problems of scirrhous gastric cancer is the development of peritoneal metastasis. In the study by Kinoshita et al., 13 of the 55 patients $(24 \%)$ had peritoneal seeding at the time of operation despite a negative laparoscopy before neo-adjuvant treatment [18]. To prevent peritoneal recurrence, hyperthermic intraoperative intraperitoneal chemotherapy (HIIC) has been shown to be beneficial in a meta-analysis [19], but this treatment is not standard owing to an increase in postoperative morbidity, and not proven superiority over systemic chemotherapy.

At this moment, the only randomized surgical trial is the Japanese JCOG 0110 on splenectomy versus splenic preservation for proximal gastric carcinoma. All other trials focus on pre-, post-, or perioperative treatment. Current accruing phase III trials are: the British ST-03 MRC trial, which is a continuation of the MAGIC trial and randomizes between perioperative ECC (epirubicin, cisplatin, capecetabine) with or without bevacizumab; the Dutch CRITICS trial, which uses the MAGIC perioperative ECC as standard therapy and randomizes between ECC or radiochemotherapy after adequate surgery; the United States intergroup CALGB 80101 trial, which randomizes between perioperative 5FU/leucovorin before and after 5-FU-based chemoradiation, and the same schedule with ECF replacing 
5-FU/leucovorin; the Italian HIIC trial, which randomizes between no postoperative treatment and HIIC after neo-adjuvant chemotherapy (EOX) and D2 surgery; and the Japanese JCOG 0501 trial, which randomizes between neo-adjuvant S1-cisplatin and surgery alone.

In conclusion, prevention could lead to a decrease of gastric cancer-related deaths. Early detection of scirrhous gastric cancer is difficult even in countries with screening programs. The maximum effect of surgery for advanced gastric cancer seems to have been reached. The use of (neo)adjuvant treatment is the only way to further improve results. Current trials with more effective drugs and/or radiotherapy are under way to evaluate such potential improvements.

\section{References}

1. Japanese Gastric Cancer Association Registration Committee, Maruyama K, Kaminishi M, Hayashi K, Isobe Y, Honda I, et al. Gastric cancer treated in 1991 in Japan: data analysis of nationwide registry. Gastric Cancer 2006;9:51-66.

2. Liu C, Russell RM. Nutrition and gastric cancer risk: an update. Nutr Rev 2008;66:237-49.

3. Uemura N, Okamoto S, Yamamoto S, Matsumura N, Yamaguchi $\mathrm{S}$, Yamakido M, et al. Helicobacter pylori infection and the development of gastric cancer. N Engl J Med 2001;345:784-9.

4. Fuccio L, Zagari RM, Minardi ME, Bazzoli F. Systematic review: Helicobacter pylori eradication for the prevention of gastric cancer. Aliment Pharmacol Ther 2007;25:133-41.

5. Fukase K, Kato M, Kikuchi S, Inoue K, Uemura N, Okamota S, et al. Effect of eradication of Helicobacter pylori on incidence of metachronous gastric carcinoma after endoscopic resection of early gastric cancer: an open-label, randomised controlled trial. Lancet 2008;372:392-7.

6. Kamangar F, Dores GM, Anderson WF. Patterns of cancer incidence, mortality, and prevalence across five continents: defining priorities to reduce cancer disparities in different geographic regions of the world. J Clin Oncol 2006;24:2137-50.

7. Hartgrink HH, van de Velde CJ, Putter H, Bonenkamp JJ, Klein Kranenbarg E, Songun I, et al. Extended lymph node dissection for gastric cancer: who may benefit? Final results of the randomized Dutch gastric cancer group trial. J Clin Oncol 2004;22: 2069-77.

8. Cuschieri A, Weeden S, Fielding J, Bancewicz J, Craven J, Joypaul $\mathrm{V}$, et al. Patient survival after D1 and D2 resections for gastric cancer: long-term results of the MRC randomized surgical trial. Surgical Co-operative Group. Br J Cancer 1999;79:1522-30.

9. Wu CW, Hsiung CA, Lo SS, Hsich MC, Chen JH, Li AF, et al. Nodal dissection for patients with gastric cancer: a randomised controlled trial. Lancet Oncol 2006;7:309-15.

10. Sasako M, Sano T, Yamamoto S, Kurakawa Y, Nashimoto A, Kurita A, et al. D2 lymphadenectomy alone or with para-aortic nodal dissection for gastric cancer. N Engl J Med 2008;359: 453-62.

11. Fiorica F, Cartei F, Enea M, Licata A, Cabibbo G, Carau B, et al. The impact of radiotherapy on survival in resectable gastric carcinoma: a meta-analysis of literature data. Cancer Treat Rev 2007;33:729-40.

12. Macdonald JS, Smalley SR, Benedetti J, Hundahl SA, Estes NC, Stemmermann GN, et al. Chemoradiotherapy after surgery compared with surgery alone for adenocarcinoma of the stomach or gastroesophageal junction. N Engl J Med 2001;345:725-30.

13. Kim S, Lim DH, Lee J, Kang WK, Macdonald JS, Park CH, et al. An observational study suggesting clinical benefit for adjuvant postoperative chemoradiation in a population of over 500 cases after gastric resection with D2 nodal dissection for adenocarcinoma of the stomach. Int J Radiat Oncol Biol Phys 2005;63: 1279-85.

14. Cunningham D, Allum WH, Stenning SP, Thompson JN, van de Velde CJ, Nicolson M, et al. Perioperative chemotherapy versus surgery alone for resectable gastroesophageal cancer. N Engl J Med 2006;355:11-20.

15. Hartgrink HH, van de Velde CJ, Putter H, Songun I, Tesselaar ME, Klein Kranenbarg E, et al. Neo-adjuvant chemotherapy for operable gastric cancer: long term results of the Dutch randomised FAMTX trial. Eur J Surg Oncol 2004;30:643-9.

16. Boige V, Pignon J, Saint-Aubert B, Lasser T, Conroy O, Bouche $\mathrm{P}$, et al. Final results of a randomized trial comparing preoperative 5-fluorouracil $(\mathrm{F}) /$ cisplatin $(\mathrm{P})$ to surgery alone in adenocarcinoma of stomach and lower esophagus (ASLE): FNLCC ACCORD07-FFCD 9703 trial. Proc Am Soc Clin Oncol 2007;25: A4510.

17. Sakuramoto S, Sasako M, Yamaguchi T, Kinoshita T, Fujii M, Nashimoto A, et al. Adjuvant chemotherapy for gastric cancer with S-1, an oral fluoropyrimidine. N Engl J Med 2007;357: 1810-20.

18. Kinoshita T, Sasako M, Sano T, Katai H, Furukawa H, Tsuburaya A, et al. Phase II trial of TS-1 for neoadjuvant chemotherapy against scirrhous gastric cancer (JCOG0002). Gastric Cancer 2009;12:37-42.

19. Yan TD, Black D, Sugarbaker PH, Zhu J, Yonemura Y, Petrou $\mathrm{G}$, et al. A systematic review and meta-analysis of the randomized controlled trials on adjuvant intraperitoneal chemotherapy for resectable gastric cancer. Ann Surg Oncol 2007;14:2702-13. 